\title{
Bullshit in a network structure: the two-sided influence of self-generated signals
}

\author{
Tomer Tuchner $^{1}\left[\right.$. Gail Gilboa-Freedman ${ }^{2}$
}

Received: 28 February 2020 / Revised: 21 July 2020 / Accepted: 23 July 2020 / Published online: 5 August 2020

(c) Springer-Verlag GmbH Austria, part of Springer Nature 2020

\begin{abstract}
In today's social network age, information flowing in networks does not derive solely from external sources; people in the network also independently generate signals. These self-generated signals may not be deliberate lies, but they may not bear any relationship with the truth, either. Following the philosopher Harry G. Frankfurt, we refer to such self-generated signals as bullshit. We present an information diffusion model that allows nodes which hold no value to spread information, capturing the diffusion of bullshit information. The presence of self-generated signals (i.e., bullshit) increases the amount of information available for transmission in the network. However, participants in the spread process respond to the existence of such self-generated information by receiving data from internal sources with caution. These two contradictory forces-the increase in information transmission on the one hand, and in suspicion on the other-result in a two-sided effect of bullshit on the total spread time. We first take a numerical approach, simulating our model on Watts-Strogatz networks and building a decision tree to characterize the effects of bullshit given different network structures. We find that increasing the rate of self-generated information may have either a monotonic or non-monotonic effect on the rumor spread time, depending on the network structure and rate of non-self-generated internal communications. Then, taking an analytical approach, we analyze the spread behavior for cliques, and identify the conditions for monotonic behavior in a 2-clique network.
\end{abstract}

Keywords Rumor spread $\cdot$ Bullshit $\cdot$ Advertising $\cdot$ Word of mouth $\cdot$ Social networks $\cdot$ Decision trees

\section{Introduction}

It is impossible for someone to lie unless he thinks he knows the truth. Producing bullshit requires no such conviction.

Harry G. Frankfurt, "On Bullshit" (Frankfurt and Wilson 2005)

In today's social media age people are bombarded with information, not all of which is trustworthy and dependable. Online social networks are fertile ground not only for liars, who use falsehood to hide the truth, but also for bullshitters,

Tomer Tuchner

tomertuc@gmail.com

Gail Gilboa-Freedman

gail.gilboa@idc.ac.il

1 Efi Arazi School of Computer Science, IDC Herzliya, P.O. Box 167, Herzliya, Israel

2 Adelson School of Entrepreneurship, IDC Herzliya, P.O. Box 167, Herzliya, Israel who don't care whether what they say is true or false. The source of bullshit information is often network-internal, generated by users who lack either access to or interest in seeking out trustworthy data. Meanwhile, users receiving transmitted information have two choices. People may continue to spread a received signal, despite not knowing whether it is true. Or people may be suspicious about the reliability of a received signal, and ignore it.

Too much bullshit information in a system leads to a lack of trust. This is a concern not only from the perspective of users, but also from the perspective of the companies providing social-network services. Freedom of speech is the fuel of social-network platforms, allowing users to pour out their thoughts, opinions, and speculations without censure. However, freedom of speech may also be the adversary of such platforms, when that freedom jeopardizes the trust people assign to the information disseminated through the platform. Without trust, people stop receiving and transmitting information, and may eventually leave the platform. Imagine an extreme case where information is generated at a high rate but immediately evaporates, without being spread at 
all. Soon, the platform will have no users. Therefore, socialnetwork firms like Facebook, Twitter, Instagram, and others have a strong interest in understanding the dynamics of information spread within networks, and the consequences of self-generated signals in a network structure.

Surprisingly, there is no clear-cut framework in the scientific literature for studying how rumors spread when each node has the capacity both to transmit and to produce signals. In particular, no existing framework allows for estimating the effects of changes in the rate of self-generated signals on rumor spread. The present study explores these dynamics.

In Tuchner and Gilboa-Freedman (2019), we proposed a rumor spread model that captures this concept of selfgenerated signals. The nodes do not merely transfer information received from other nodes, but can also generate new information. Accordingly, the information flow process now incorporates a dimension of suspicion. The Covid-19 pandemic, ongoing as of this writing, offers a fitting example. In this situation, people are eager for information about the values of different variables - for example, official mortality rates from the disease. Our model assumes that the value of this variable at any time is available from some trustworthy external source (e.g., national health authorities), but that this information requires some effort to access and check. Our model also assumes that there is internal diffusion of information about this value within the network, from one person to another, and that this internal diffusion may represent either the real value, or false values invented by noninformed individuals. (We recognize that in the case of the Covid-19 pandemic there is debate about the methods used by official bodies to record statistics such as infection and mortality rates. However, for the purposes of this example, we note that official statistics serve as a basis for policy making and citizens' personal decisions, and therefore knowing the true value of official statistics is important.)

The current paper uses the rumor spread model developed by Tuchner and Gilboa-Freedman (2019) to further investigate the influence of these self-generated signals. The high-level goal of both papers is to explore the influence of self-generated signals on the properties of the spread process given a certain network structure. The contribution of the current study over the previous paper is twofold. (1) We describe here a simpler version of the model, where nodes are in one of two states (informed vs. non-informed), without distinguishing between pure and non-pure informed states as in Tuchner and Gilboa-Freedman (2019). (2) In this study we deepen our examination into how self-generated signals influence a particular property of networks, which we call the existence of a turning point. We do this by extending our earlier numerical study and adding an analytical one.

From the perspective of the manager of a social network company, it is important to evaluate whether bullshit information stimulates or depresses activity on the platform.
We use our model to examine an equivalent question: will self-generated signals accelerate or slow the rate of propagation? The answer is not straightforward, as the dynamics of self-generated signals involve two opposing forces: on the one hand, spontaneous signals increase the aggregate amount of information produced; on the other hand, the existence of spontaneous signals increases suspicion towards any received information. The goal of our research is therefore to identify which force will dominate the other, given particular model parameters. Interestingly, we find that for some parameters, each force may dominate at different times: increasing the rate of bullshit production up to some threshold will accelerate the rate of propagation, but increasing the bullshit production rate beyond that threshold will slow the propagation rate. This threshold is what we call a turning point.

We next describe our "bullshit model" (Tuchner and Gilboa-Freedman 2019). The bullshit model extends the independent cascade model by adding diffusion processes that are sourced at internal resources (i.e., generated by noninformed nodes). (1) At the start of the process, all nodes are non-informed. (2) Once the process begins, a signal is transmitted by an external source. (3) Additionally, signals can be transmitted by any node, whether informed or not, independently of the history of the process. (4) Nodes may accept received signals and become active, but signals generated internally are accepted with caution. (5) This process runs until a predefined proportion of the nodes are activated.

To analyze our model, we start by simulating the rumor spread process on two graphs: a Facebook sample graph and a random graph. In general networks, the spread of a rumor is a complex process involving a large number of actions taken by many interacting entities. For this reason, our approach combines a numerical study of simulation results over different parameters with an analytical study of simple networks.

Our numerical study sheds light on how the rate at which bullshit spreads in a network influences the time required for the predefined percent of nodes to become informed (i.e., for those nodes to possess information, regardless of whether that information is generated by a reliable external source or an unreliable internal source). Specifically, we examine whether the rate of spread is monotonic or non-monotonic in large network structures, using combinations of the model parameters. We use a decision tree approach to characterize the network structures in which encouraging bullshit has a non-monotonic effect-that is, where increasing the bullshit rate up to some level makes the network more active, but increasing this rate further has the opposite effect. Our decision tree shows that whether the graph is characterized by such opposing behaviors for different rates of bullshit spread is closely related to the sparseness of the graph and the rate at which information from informed nodes is transmitted. 
Our analytical study considers the special case of a 2-clique network, computing the expected time until both nodes become informed. For this fundamental framework, we examine how the choice of model parameters affects whether increasing the rate of self-generated signals has a monotonic or non-monotonic effect. We also present a framework for analyzing the more complex case of a general clique. The results shed light on how to use our methodology to analyze other network topologies (specifically, by solving a set of transition equations and achieving the expected number of iterations needed to complete the rumor spread, and then calculating the partial derivative of this value with respect to the self-generated signals transmission rate).

\section{Related work}

In today's post-truth era (Keyes 2004), social media technologies increasingly govern our access to news (Gottfried and Shearer 2016), and our response to everything from natural disasters (Mendoza et al. 2010; Gupta et al. 2013) to terrorist attacks (Starbird et al. 2014). Hence, there is increasing interest within the scientific literature in the concept of "fake news," and specifically the epidemiology (Kucharski 2016), detection (Conroy et al. 2015), and impact of false or misleading stories. The "fake news" phenomenon has the potential to influence attitudes towards journalistic objectivity (Marchi 2012), and may impose real costs on society and politics (Allcott and Matthew 2017). From a corporate point of view, false stories have potential to damage a firm's or brand's image and propel firms into financial disaster (Hayes and Kotwica 2013). Of course, false stories have always existed - but the ability of social media platforms to spread such narratives rapidly and aggressively gives the question new importance. One recent study focusing on the social network Twitter found that fake news "diffused significantly farther, faster, deeper, and more broadly than the truth in all categories of information" (Vosoughi et al. 2018).

Works on the spread of fake news within networks have largely studied ways to identify untrustworthy information (Dhar et al. 2016), and to restrict and contain it (Budak et al. 2011; Fan et al. 2014). Such works generally consider the factual accuracy of a rumor, and look for ways to identify untruthful messages of various types, such as hoaxes, irony or propaganda (Wang et al. 2019). In our model, we capture the creation of fake information, but we consider only the originator of the data - an external source or an internal noninformed node-to classify the information as reliable or unreliable, without examining the content of the message.

Reliability in general, and rumor reliability in particular, is a vast research topic spanning multiple disciplines, and a central concept in theories of decision-making (Savage 1951; Simon 1960; Wedgwood 2002; Habib et al.
2019), cooperation (Fehr and Fischbacher 2003), communication (Shannon 1948), viral marketing (Jurvetson 2000; Krider and Weinberg 1998), and markets (Bikhchandani et al. 1992). The study of reliability commonly draws on network models, which often use the term reliability for the probability that the proportion of informed individuals exceeds a certain value. Some network models associate reliability with social cohesion (Wasserman and Faust 1994). Modern network models define reliability as the probability of data transmission from one element to another (Girvan and Newman 2002), or in other words, the probability that a given node will be informed.

Our model follows previous work in probability theory on interacting particle systems (Liggett 2012). We formulate the simplest extension of an independent cascade model (Kempe et al. 2003), a type of model that has been investigated in the context of marketing and word-ofmouth processes (Krider and Weinberg 1998; Goldenberg et al. 2001). The dynamics at the node level follow a predefined scheme of response probabilities and are a function of the state of the nodes with which the particle interacts, as described by Holland (1995). Our contribution is in considering richer dynamics for these interactions, specifically the possibility of activation by noninformed nodes. Unlike existing models that describe the transmission of unreliable information (Lazer et al. 2018), our model assumes not only that nodes transfer unreliable information, but also that information may be generated by nodes which have not received information regarding the rumor from an external source. As in other studies (Gilboa-Freedman and Smorodinsky 2020), the probability that a node will spread such signals onward within the graph is dependent on the node's state, with a scheme defined by a signal matrix.

In a recent study considering the dynamics of reinvention and its effect on information diffusion, Koren et al. (2014) found that network topology plays a key role in critical mass formation and diffusion properties. Our numerical results strengthen this finding by showing a strong link between the sparseness of a network and diffusion properties in a setup involving bullshit information. We also show that in the same topology, the influence of self-generated signals can be monotonic or non-monotonic, sometimes speeding up and sometimes slowing down the spread time. Our analytical study examines these dynamics in a 2-clique network.

We simulate the rumor spread process on a Facebook sample graph taken from the SNAP project (Leskovec 2012), on a random graph, and on a series of synthetic Watts-Strogatz networks (Watts and Strogatz 1998). Like other studies in the literature on rumor spread online (Cuypere et al. 2016), we also consider a mid-sized network (500 nodes). The intuition behind this is that opinions and rumors often spread within a particular online community which is not 
that large-for example, people contributing to an online forum, or people tweeting and re-tweeting some hashtag.

The complex interactions in the network generate aggregated patterns which are hard to predict (Waldrop 1993). Hence, we analyze the simulation results by organizing them in a decision tree (Quinlan 1986). Decision trees are widely used in machine learning (Kotsiantis et al. 2007), with the purpose of predicting a target value (a class) from some input features. To build the decision tree, a modeler uses a data set that includes a list of measurable properties (features), one of which is the target. Decision trees are among the most popular predictive models-for a survey see Safavian and Landgrebe (1991). They are also used as descriptive tools (Delen et al. 2013).

\section{Objectives}

Self-generated signals have two contradictory effects. On the one hand, they increase the amount of information transmitted between neighbors in any single iteration. On the other hand, the presence of self-generated signals adds an element of suspicion, making it less probable that any given node will accept the information. If the first effect is dominant over the second, then increasing the "bullshit level" (the transmission rate of self-generated signals) stimulates network activity, and rumors spread faster. If the second effect is dominant, increasing the bullshit level depresses activity, and rumors spread more slowly.

Interestingly, some network setups exhibit both effects. In these cases, increasing the transmission rate of selfgenerated signals has an inconsistent influence: for certain ranges of the transmission rate, increasing the transmission rate causes rumors to spread more quickly, while for other ranges, increasing the transmission rate causes rumors to spread more slowly. The present study aims to classify network setups by their tendency to exhibit this interesting and paradoxical property, which we call existence of a turning point.

From the perspective of social-network firms, it is important to know how the transmission of bullshit information will affect activity on the platform, and in particular whether it will stimulate or depress such activity. Platform managers can then use this information to better manage activity on the platform (e.g., through policies designed to educate or punish users who transmit bullshit). Platform managers also need to know whether the influence of transmitting bullshit is consistent for different bullshit levels, as the same policy may be efficient for some bullshit levels but not for others.

We use computational simulations to study general random networks, and analytical methods to study the fundamental case of a 2-clique network.

\section{Model}

Let us have a network $G=(V, E)$ with nodes $V=\left(v_{1}, v_{2}, \ldots, v_{n}\right)$. The state of the model in a specific iteration is

$S=\left(s_{1}, s_{2}, \ldots, s_{n}\right)$

where

$s_{i}= \begin{cases}1, & \text { if } v_{i} \text { is informed } \\ 0, & \text { if } v_{i} \text { is non-informed }\end{cases}$

In each iteration, the model is also described by

$T=\left(t_{1}, t_{2}, \ldots, t_{n}\right)$

where

$t_{i}= \begin{cases}1, & \text { if } v_{i} \text { transmits a signal } \\ 0, & \text { if } v_{i} \text { does not transmit a signal. }\end{cases}$

The probability that a node will transmit a signal is determined by its state. The relationship between the two is described in Table 1.

The probability that $v_{i}$ will transmit a signal in a given iteration is

$s_{i} P+\overline{s_{i}} Q$

where

$\overline{s_{i}}= \begin{cases}1, & \text { if } s_{i}=0 \\ 0, & \text { if } s_{i}=1\end{cases}$

We highlight the fact that the model ascribes a positive probability $Q$ to transmission (generation) of signals by noninformed nodes. To put this in a real-world context, consider again a public event where information about some variable is difficult to come by. In such situations, bullshitters spread information that is self-generated (not held), including fictitious values of the variable of interest. The early stages of the Covid-19 pandemic in February and March 2020 offer a prime example: infection rates, hospitalization rates, and mortality rates were all uncertain and changing rapidly, opening the door wide to hunches, predictions, and stabs in

Table 1 The table describes the probability that a node will transmit a signal in a specific iteration

\begin{tabular}{lll}
\hline & Transmits & Does not transmit \\
\hline Informed & $P$ & $1-P$ \\
Non-informed & $Q$ & $1-Q$ \\
\hline
\end{tabular}

The rows represent the state of the node (i.e., whether or not it is informed). The columns represent the probability that the node will transmit a signal 
the dark that were then passed on as "news." Other examples might include casualty figures following a military attack or natural disaster.

In each iteration, non-informed nodes can become informed, a shift known as activation. A node can be activated either by an external signal, or by an internal signal from a neighboring node.

Activation by an external signal occurs with probability $\alpha$ for each node independently. This represents the probability of learning the variable of interest-for example, the number of casualties following a military attack-from a legitimate resource like a newspaper. Activation by an internal signal follows the following scheme: for each noninformed node $v$ where a neighboring node $u$ is transmitting a signal, $\frac{P}{P+Q}$ is the probability that $v$ accepts the signal being transmitted, thereby becoming informed. This probability serves as a "trust factor." The trust factor is used to represent nodes' suspicion towards self-generated signals. This captures the likelihood of signals coming from an informed node, from the perspective of someone with no prior knowledge about the distribution of informed vs. non-informed nodes in the network. Returning to our example, the trust factor represents the probability that a user of a social media platform will accept a casualty figure provided by a friend (rather than a legitimate media resource, like the evening news).

In total, activation of $v_{i}$ (when non-informed) in a specific iteration occurs with probability

$\alpha+(1-\alpha)\left\{1-\prod_{\left(v_{j}, v_{i}\right) \in E}\left[1-\frac{P}{P+Q}\left(s_{j} P+\overline{s_{j}} Q\right)\right]\right\}$.

The process is fully described in Algorithm 1.

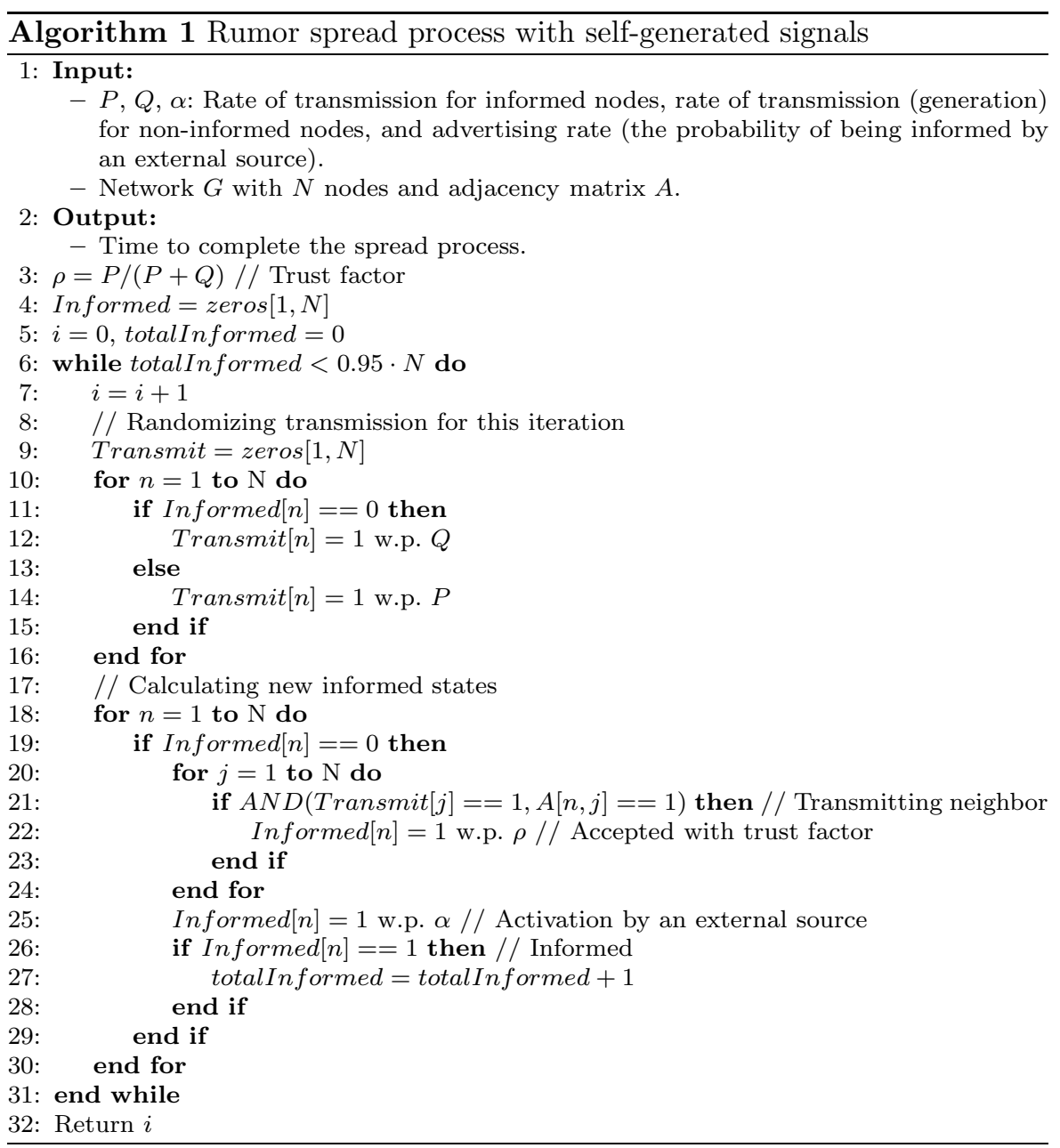




\section{Useful definitions}

We provide some definitions that are useful throughout our analysis.

Definition 1 Model Setup: A model setup of the rumor spread model is a 4-tuple $(G, P, Q, \alpha)$ containing, respectively, the network within which the rumor spreads, the rate of transmission by informed (i.e., activated) nodes, the rate of transmission by non-informed nodes (i.e., self-generated signals), and the rate of advertising by an external source.

Definition 2 Basic Setup: A basic setup of the rumor spread model is a 3-tuple $(G, P, \alpha)$ comprising the set of all model setups $(G, P, Q, \alpha)$ where $Q \in[0,1]$.

Definition 3 Spread Time Function: For any set of model setups $M$, a spread time function is a function $T: M \rightarrow \mathbb{R}^{+}$.

The spread time function defined by definition 3 represents the time to completion of the rumor spread process for each setup. This can be retrieved in several different ways, depending on the context. In our study, it is sometimes computed as an average of several spread simulations for the same model setup (as in Sects. 6, 7), and sometimes retrieved analytically as the expected completion time for a given model setup (as in Sect. 8.2).

Now, let us define the property we wish to examine: existence of a turning point.

Definition 4 Existence of a Turning Point: Given a spread time function $T$, we say that a basic setup $(G, P, \alpha)$ satisfies the existence of a turning point property if there exists $Q_{1}<Q_{2}<Q_{3}$ such that $T\left(G, P, Q_{1}, \alpha\right)>T\left(G, P, Q_{2}, \alpha\right)<T\left(G, P, Q_{3}, \alpha\right)$.

Notice that the above definition for existence of a turning point (Definition 4) formalizes the objectives described in Sect. 3. Our goal is to classify basic network setups by whether or not increasing the transmission rate of self-generated signals has different influence for different ranges- that is, to identify basic setups where there exists a turning point.

\section{Example: Facebook sample and a random network}

We start by simulating our model on a Facebook sample graph and on an Erdős-Rényi random graph. The two graphs are equivalent in the number of their nodes (4039) and their average degree (approx. 44). However, the two differ in their

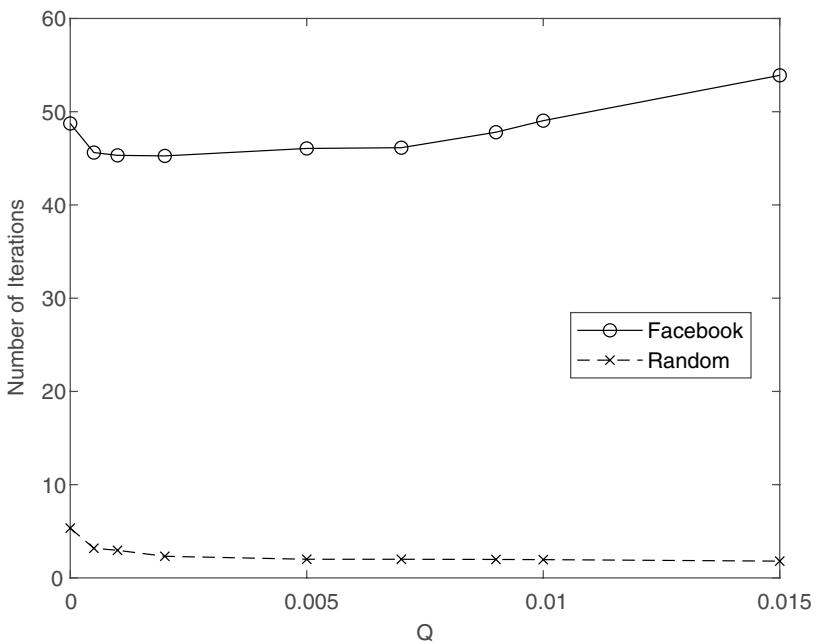

Fig. 1 Rumor spread time as a function of the probability of noninformed nodes spreading self-generated signals. The figure plots the number of iterations required for $95 \%$ of the nodes to become informed as a function of $Q$ when $P$ and $\alpha$ are fixed to 0.01 . Each result is an average of 20 simulations over the same setup

topology (e.g., the distribution of the node degrees). The Facebook sample graph was taken from the SNAP project (Leskovec 2012). The equivalent random graph was generated by a computer program in Matlab.

Figure 1 displays the number of iterations needed for $95 \%$ of nodes to become informed against $Q$, where $P$ and $\alpha$ are fixed at 0.01 , for the two networks (i.e., the Facebook sample and the random graph). Each result is an average of 20 simulations over the same setup. The figure provides an example of a basic setup (see Definition 2) characterized by the existence of a turning point (Facebook), and an example of a basic setup that does not exhibit this property (the equivalent random graph). The Facebook network shows a range of $Q$ values where the spread time decreases with an increase in the transmission rate of self-generated signals (at the far left of the graph, where $Q$ is low). However, for larger $Q$ values the spread time increases (i.e., the rumor spread process slows down). The random network exhibits monotonic decreasing behavior as $Q$ rises. Thus, Fig. 1 demonstrates that increasing the rate of self-generated signals may or may not slow down the spread of the rumor, depending on the network's topology.

There is a magnitude difference between the rates of spread in the random graph vs. the Facebook sample graph. However, it is the qualitative behavior of the spreads in these networks (monotonic or not) that is important in the present study. The random graph parameters were set so the two graphs would have the same number of nodes and the same average degree. It is shown that whether a turning point exists is not trivial, and is influenced by the topology of the network. 


\section{Numerical study}

We simulate the rumor spread process as described by the model for a variety of model parameters and network structures. We then analyze the simulation results, using decision trees to classify the simulations by their properties. The simulations were conducted in a Matlab environment. The code used for the simulations is available online (Tuchner and Gilboa-Freedman 2020c).

\subsection{Preparation of networks}

We wrote a Matlab program to generate synthetic networks using an algorithm from Watts and Strogatz (Watts and Strogatz 1998). These networks have 500 nodes and vary on the parameters $K$ and $\beta$, which describe, respectively, the ratio between edges and nodes in the graph, and how close the graph is to an Erdős-Rényi random graph, where $\beta=1$ implies a random network and $\beta<1$ displays properties of a small-world structure (closer to a social network).

\subsection{Simulations}

Combinations of model parameters $(\alpha, P, Q)$ and network parameters $(K, \beta)$ were considered in a full factorial design experiment. We set $Q$ - the probability of a non-informed node transmitting a self-generated signal- to be lower than $P$, under the reasonable assumption that being informed about a rumor should not reduce the probability of spreading information.
Each of the five input variable parameters was manipulated to produce a variety of spread process simulations. For each set of parameters we ran 20 simulations and calculated their mean results. In total we examined 41,400 sets of parameters (no $\alpha=Q=0$ ), in each case running the rumor spread process until $95 \%$ of the nodes were informed.

The parameter ranges were set as follows:

1. $K$-Half the average degree $\quad 3,5,8,10,15,20$

2. $\beta$-Randomness of the graph $0.5,0.8,1$

3. $\alpha$-Advertising rate $\quad 0-0.1$ (steps of 0.005 )

4. $P$-Informed rate $\quad 0.01-0.1$ (steps of 0.01 )

5. $Q$-Non-informed rate $\quad 0-P(10$ constant steps $)$

where advertising rate refers to activation by an external source; informed rate refers to transmission by an informed node; and non-informed rate refers to transmission (generation) by a non-informed node.

\subsection{Analysis and results}

After generating all possible outcomes over the set of simulation parameters, we analyzed the influence of the parameters on the behavior of the rumor spread. For this purpose, we took the decision tree approach to elicit relevant observations. The simulation results served as input data for training a decision tree-i.e., set of rules organized in a hierarchical structure that could serve as a predictive model for the relevant measure. The tree was constructed using a Python program available in GitHub (Tuchner and Gilboa-Freedman 2020a).

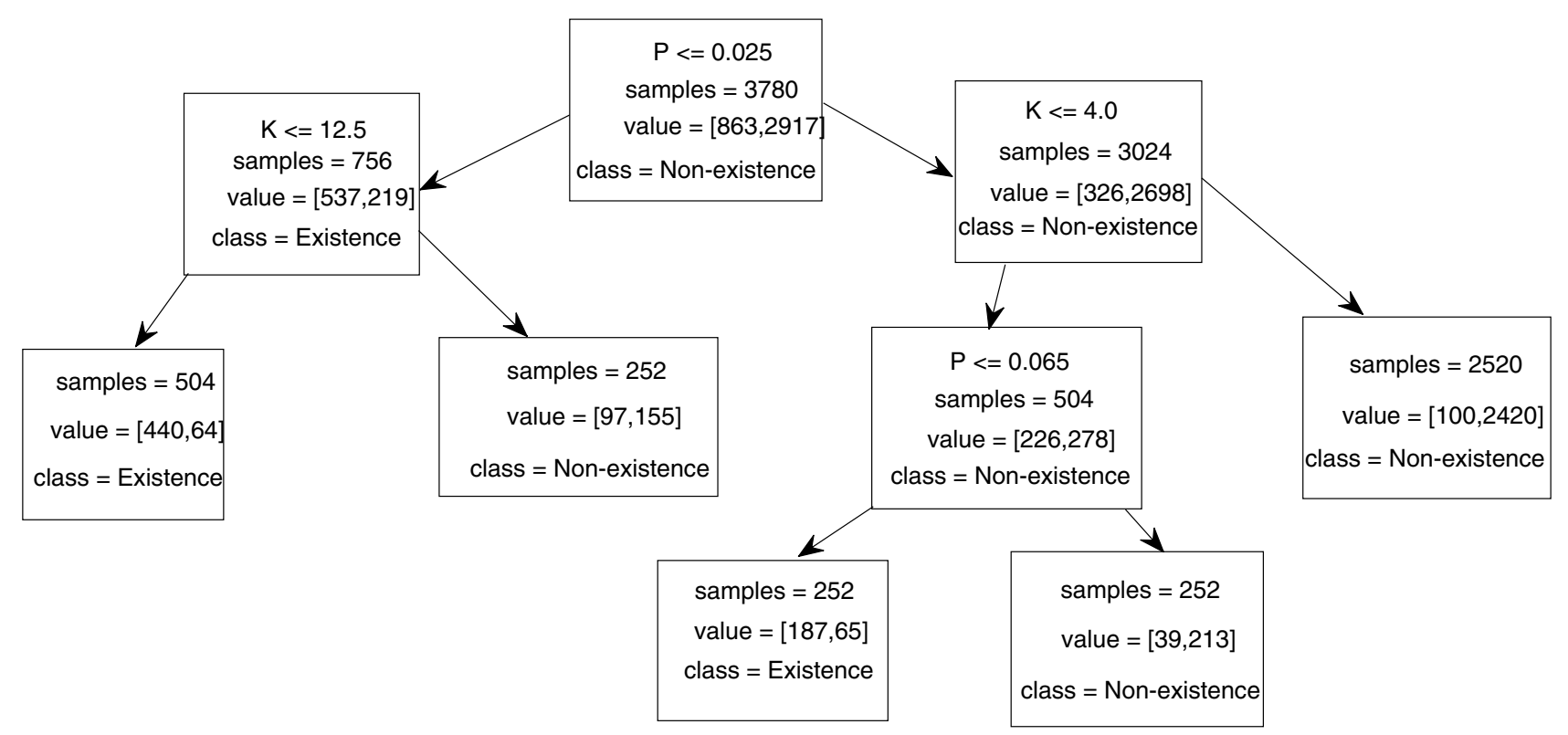

Fig. 2 Decision tree to predict existence of a turning point for a given basic setup. For each leaf, the tree specifies the number of samples that were sorted into this leaf over the categories of the target; their distribution in terms of how many samples fall in each category; and the prediction assigned to this leaf. For each internal node (not a leaf), the graph also specifies the splitting criterion 
A decision tree specifies the properties of its nodes. Specifically, for each leaf (a node with no arrow issuing from it), the tree specifies the number of samples sorted into this leaf over the categories of the target; their distribution in terms of how many samples fall in each category; and the prediction assigned to this leaf. For each internal node (i.e., all nodes that are not leaves), the graph specifies the aforementioned properties, and also the splitting criterion.

We test for the existence of a turning point, defined formally by Definition 4. Our analysis yields the decision tree in Fig. 2, from which we can deduce that turning points usually appear when the network is sparse and internal communication between informed nodes is low.

More precisely, the figure shows two branches that predict the existence of a turning point. In both, the transmission rate by informed nodes and the average degree are bounded from above. In the first branch, the informed rate is very low $(P \in\{0.01,0.02\})$ and the network is fairly sparse ( $K \in\{3,5,8,10\})$. The samples that fall into this branch have a high tendency to demonstrate a turning point $(87 \%$ of the samples, as compared to $23 \%$ of the total set of simulations). In the second branch, the network is even more sparse $(K=3)$ and the transmission rate by informed nodes is at its lower values $(P \in\{0.01,0.02,0.03,0.04,0.05\})$. In that case, $74 \%$ of the samples demonstrate a turning point.

\section{Analytical study}

The goal of our analytical study is to identify an analytical condition whereby a network exhibits a turning point (see Definition 4).

As we already observed in our numerical study, and as we saw when comparing the Facebook and random graphs in Sect. 6, how the model parameters influence the rumor spread process is complex. Thus, analytically examining the spread behavior is difficult. For this reason we apply our analytical study to a very basic system. The insights derived from this study can then be applied to similar phenomena in more complex systems.

\subsection{The case of an $n$-clique}

To analyze rumor spread in a model that incorporates self-generated signals, we consider an $n$-clique graph. Specifically, we focus on how the advertising rate, $\alpha$, and the transmission rate by informed nodes, $P$, determine the existence (or non-existence) of a turning point. To motivate our analysis, we consider once again the manager of a social-network firm who aims to evaluate how the presence of bullshit information affects activity on his platform. One key area of interest is the influence of bullshit on activity for a given network topology. $n$-clique networks were chosen for this examination as fundamental topologies, that allow stripping the analysis of characteristics that are unrelated to propagation rates.

Let $\left\{X_{i} \mid i=0,1,2, \ldots, n\right\}$ be a Markov chain where state $X_{i}$ represents a state in which $i$ nodes are informed.

We define a transition matrix $A$, where $A_{i, j}$ is the probability that the process will transition from state $X_{j}$ (where exactly $j$ nodes are informed) to state $X_{i}$ in the next iteration. Put differently, $A_{i, j}$ is the probability that when exactly $j$ nodes are informed, $i-j$ nodes are activated in a single iteration.

$A_{i, j}$ is determined by combinations of the values of the following counters:

- $K_{1}$ counts the number of nodes that transmit a signal in a given iteration out of the population of $j$ informed nodes.

- $K_{2}$ counts the number of nodes that transmit a signal in a given iteration out of the population of $n-j$ non-informed nodes.

- $K_{3}$ counts the number of non-informed nodes that both become activated and transmit a signal in a given iteration. Its range is circumscribed by the counters described in Table 2, which must be positive.

Table 2 presents the counters of each type for non-informed nodes: whether or not they are activated, crossed by whether or not they transmit a signal.

The following equation calculates $A_{i, j}$ by summing the probabilities of all possible combinations of $K_{1}, K_{2}$, and $K_{3}$ :

$$
\begin{aligned}
& A_{i, j}(n, \alpha, P, Q) \\
& =\left(\begin{array}{c}
n-j \\
i-j
\end{array}\right) \sum_{K_{1}=0}^{j} \sum_{K_{2}=0}^{n-j} \sum_{K_{3}=\max \left(0, K_{2}-(n-i)\right)}^{\min \left(K_{2}, i-j\right)}\left(\begin{array}{c}
j \\
K_{1}
\end{array}\right)\left(\begin{array}{c}
n-j \\
K_{2}
\end{array}\right) . \\
& \cdot\left(\begin{array}{c}
\min \left(K_{2}, i-j\right) \\
K_{3}
\end{array}\right) \cdot P^{K_{1}} \cdot(1-P)^{j-K_{1}} . \\
& \text { - } Q^{K_{2}} \cdot(1-Q)^{(n-j)-K_{2}} \text {. } \\
& \left\{\alpha+(1-\alpha)\left[1-\left(1-\frac{P}{P+Q}\right)^{K_{1}+K_{2}-1}\right]\right\}^{K_{3}} \text {. } \\
& \left\{\alpha+(1-\alpha)\left[1-\left(1-\frac{P}{P+Q}\right)^{K_{1}+K_{2}}\right]\right\}^{(i-j)-K_{3}} \text {. } \\
& {\left[(1-\alpha)\left(1-\frac{P}{P+Q}\right)^{K_{1}+K_{2}-1}\right]^{K_{2}-K_{3}} \text {. }} \\
& {\left[(1-\alpha)\left(1-\frac{P}{P+Q}\right)^{K_{1}+K_{2}}\right]^{(n-i)-\left(K_{2}-K_{3}\right)}}
\end{aligned}
$$

To compute the state transition probability, we first need to choose the $(i-j)$ activated nodes out of possible $(n-j)$ 
Table 2 Notational description of the counters for non-informed nodes, based on whether they transmit or do not transmit, crossed by whether they are activated or remain non-informed

\begin{tabular}{lll}
\hline & Transmits & Does not transmit \\
\hline Activated & $K_{3}$ & $(i-j)-K_{3}$ \\
Not activated & $K_{2}-K_{3}$ & $(n-i)-\left(K_{2}-K_{3}\right)$ \\
\hline
\end{tabular}

non-informed ones. Once we know $K_{1}, K_{2}$ and $K_{3}$, we need to choose which $K_{1}$ informed nodes will be transmitting out of possible $j$ informed nodes; which $K_{2}$ non-informed nodes will be transmitting out of possible $(n-j)$ non-informed nodes; and which are the $K_{3}$ newly activated transmitting nodes out of possible $K_{2}$ transmitting non-informed nodes and $(i-j)$ newly activated nodes.

Note that $K_{3}$ should be at least $K_{2}-(n-i)$-so that the number of not-activated not-transmitting nodes $\left((n-i)-\left(K_{2}-K_{3}\right)\right.$, see table 2$)$ will be nonnegative, and should be lower than both total transmitting non-informed nodes and total newly activated nodes.

Now we compute the probability for assumed transmission state of the system: we need $K_{1}$ informed nodes to transmit with probability $P, j-K_{1}$ informed nodes to not transmit with probability $(1-P), K_{2}$ non-informed nodes to transmit with probability $Q$, and $(n-j)-K_{2}$ non-informed nodes to not transmit with probability $(1-Q)$.

Regarding activation, we need $K_{3}$ non-informed transmitting nodes to be activated - they will be activated either by an external source or by accepting any of their neighbors' signals. Since the network is a clique, for some non-infomed node $v$, all transmitting nodes (except of $v$ itself) might be the ones activating $v$, in total $K_{1}+K_{2}-1$ such transmitting neighbors. For the $(i-j)-K_{3}$ activated non-transmitting nodes, probability of activation will be the same, apart from the fact that the node is not included in the list of transmitting nodes, so there are $K_{1}+K_{2}$ transmitting neighbors. For non-activated nodes, we'll need both the external source to not transmit to the node, and for it not to accept the signal of any transmitting neighbor, $K_{1}+K_{2}-1$ or $K_{1}+K_{2}$ such transmitting neighbors, depending on whether the non-activated node transmits or not, respectively.

Let $e_{i}$ denote the expected time (number of iterations) until the entire graph is informed, given the initial state $X_{i}$. Our analysis focuses on the process by which all nodes become activated from an initial state where information is held only by an external source. That is, we analyze $e_{0}$.

We are most interested in the influence of the parameter $Q$ on $e_{0}$. As in the numerical study above, we aim to classify the basic model setups (see Definition 2) into two classes. The first class contains models where the influence of $Q$ on $e_{0}$ is monotonic ( $e_{0}$ is either increasing with $Q$ for all $Q \in[0,1]$, or decreasing with $Q$ for all $Q \in[0,1])$. The

second, complementary class of models, contains models where the influence of $Q$ on $e_{0}$ is non-monotonic ( $e_{0}$ is increasing with $Q$ for some but not all values of $Q \in[0,1]$ ).

The second class of models demonstrates the existence of a turning point (see Definition 4). In what follows (Sect. 8.2), we analyze the turning point property in the special case of a 2-clique network.

\subsection{The case of a 2-clique network}

We consider a 2-clique setup as the most fundamental scenario where external communication competes with internal communication. For this setup, we achieve a closed-form solution to describe the constraints for $(\alpha, P)$, where basic communication between two people who know each other demonstrates the existence of a turning point as discussed in this article. Note that even for such a simple case, it is not straightforward to identify the analytical condition for whether a turning point exists in a given basic setup.

The equations representing the probability of transition from a state $X_{j}$ (exactly $j$ nodes are informed) to a state $X_{i}$ (exactly $i$ nodes are informed) in a single iteration are shown in Table 3. For clarity, we use the notations $H$ and $B$ to represent the probability of activating a non-informed node when the other node is informed (and the transmitted signal is Held by the transmitting node from previous iterations) or noninformed (and the transmitted signal is Bullshit), respectively. The fact that the probabilities shown in Table 3 are identical to the probabilities achieved by substituting $n=2$ in Equation 2 was verified and excluded for triviality.

More explicitly, $H$ and $B$ are:

$H=\alpha+(1-\alpha) P \frac{P}{P+Q}$

$B=\alpha+(1-\alpha) Q \frac{P}{P+Q}$.

We wish to express $e_{i}$ using a recurrence relation. When in state $X_{i}$, we can move to any state $X_{j}$ in the chain (adding one iteration to the total time needed to inform all nodes) with probability $A_{j, i}$, and then the remaining expected time to completion will be $e_{j}$. This yields the following relation:

Table $3 A_{i, j}(2, \alpha, P, Q)$. Transition probabilities in a 2-clique network as a function of the probabilities $H, B$ that a single node will be activated when the other node is informed or non-informed, respectively

\begin{tabular}{llll}
\hline & $j=0$ & $j=1$ & $j=2$ \\
\hline$i=0$ & $(1-B)^{2}$ & 0 & 0 \\
$i=1$ & $2 B(1-B)$ & $1-H$ & 0 \\
$i=2$ & $B^{2}$ & $H$ & 1 \\
\hline
\end{tabular}

The rows represent the state the system moves to, and the columns represent the current state 
$e_{i}=1+\sum e_{j} A_{j, i}$

We want to solve the following set of linear equations:

$e_{0}=1+e_{0} A_{0,0}+e_{1} A_{1,0}+e_{2} A_{2,0}$

$e_{1}=1+e_{0} A_{0,1}+e_{1} A_{1,1}+e_{2} A_{2,1}$

$e_{2}=1+e_{0} A_{0,2}+e_{1} A_{1,2}+e_{2} A_{2,2}$

By definition, $e_{2}=0$. Substituting this, we get:

$e_{0}=1+e_{0}(1-B)^{2}+e_{1} \cdot 2 B(1-B)$

$e_{1}=1+e_{1}(1-H)$.

Solving the equations above, we get $e_{1}=\frac{1}{H}$, and

$e_{0}=\frac{H+2 B(1-B)}{H B(2-B)}$.

We are interested in whether or not the network will be characterized by existence of a turning point. Hence, we want to know for which set of parameters $(P, \alpha)$ the partial derivative $\partial e_{0} / \partial Q$ changes its sign, for example from negative values (increasing $Q$ results in a faster spread) to positive values (increasing $Q$ results in a slower spread due to suspicion). To investigate the sign of $\partial e_{0} / \partial Q$, let us recall the formula $\left(\frac{f}{g}\right)^{\prime}=\frac{f^{\prime} g-g^{\prime} f}{g^{2}}$. As the denominator does not affect the sign, let us inspect only the numerator.

We calculate the numerator using Wolfram Mathematica (Tuchner and Gilboa-Freedman 2020d). Multiplying the numerator by $(P+Q)^{6}$ (which is also always positive), we obtain:

$$
\begin{aligned}
\operatorname{Sgn}(Q)= & -2(\alpha-1) P^{2}\left\{\alpha^{4}(P+Q-P Q)^{4}\right. \\
& +\alpha^{2}(-P-Q+P Q) . \\
& {\left[P^{3}\left(-1-3 P+3 P^{2}\right)+P^{2}(7 P-3) Q\right.} \\
& +P\left(-3+23 P-18 P^{2}\right) Q^{2} \\
& \left.+\left(-1+13 P-15 P^{2}+6 P^{3}\right) Q^{3}\right] \\
& -\alpha^{3}(-P-Q+P Q)\left[P^{3}(-3-P\right. \\
& \left.+P^{2}\right)+9(-1+P) P^{2} Q-3\left(-P+P^{2}\right) \\
& (-3+4 P) Q^{2}+\left(-3+11 P-11 P^{2}\right. \\
& \left.\left.+4 P^{3}\right) Q^{3}\right]+P^{2}\left[-P^{4}+(-2+P) P^{3} Q\right. \\
& +P^{2} Q^{2}-4(-1+P) \cdot P Q^{3}+(-2+P) \\
& \left.(-1+P) Q^{4}\right]+\alpha P\left[-3 P^{5}(-1+Q)\right. \\
& +2 P(5-7 Q) \cdot Q^{3}+4 Q^{4}+P^{4}(-2+6 Q) \\
& +P^{2} Q^{2}\left(6-28 Q+13 Q^{2}\right) \\
& \left.\left.-P^{3} Q\left(2+11 Q-16 Q^{2}+4 Q^{3}\right)\right]\right\} .
\end{aligned}
$$

As we are only interested in knowing in which basic setups $\partial e_{0} / \partial Q$ changes its sign, we use Wolfram Mathematica

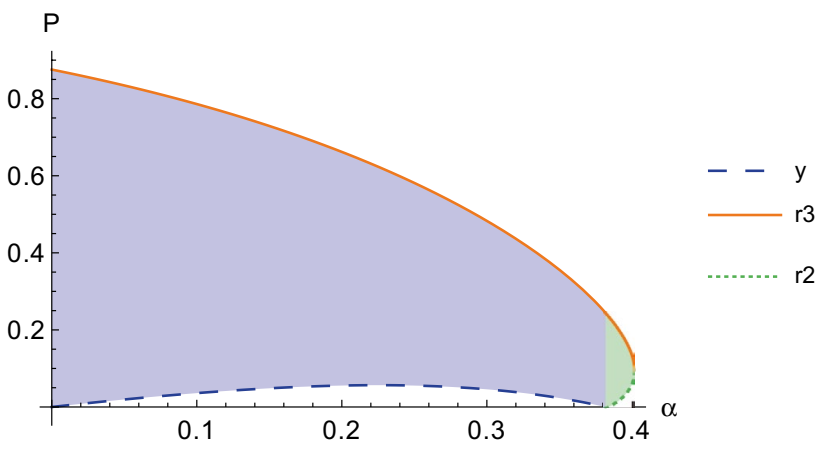

Fig. 3 Values of $(P, \alpha)$ for which a 2-clique exhibits a turning point are shown in the filled area between the lines

to check when $\operatorname{Sgn}(Q)=0$ under the condition that $\alpha, P, Q \in(0,1)$. We get the following full analytical solution:

$$
\begin{aligned}
(\operatorname{Sgn}(Q)==0) \Longleftrightarrow & \\
& {\left[\left(0<\alpha \leq \frac{1}{2}(3-\sqrt{5}) \approx 0.38\right) \wedge\left(y(\alpha)<P<r_{3}(\alpha)\right)\right] \bigvee } \\
& {\left[\left(\frac{1}{2}(3-\sqrt{5})<\alpha<0.401 \ldots\right) \wedge\left(r_{2}(\alpha)<P<r_{3}(\alpha)\right)\right] }
\end{aligned}
$$

where

$y(\alpha)=\frac{-2 \alpha+\alpha^{2}}{2(-1+\alpha)^{2}}+\frac{1}{2} \sqrt{\frac{8 \alpha^{2}-20 \alpha^{3}+17 \alpha^{4}-4 \alpha^{5}}{(-1+\alpha)^{4}}}$.

And $r_{2}(\alpha), r_{3}(\alpha)$ are the second and third roots (respectively) of the equation

$$
\begin{aligned}
\left(\alpha^{2}-\right. & \left.3 \alpha^{3}+\alpha^{4}\right)+\left(4 \alpha-10 \alpha^{2}+2 \alpha^{3}\right) x \\
& +\left(2-4 \alpha-5 \alpha^{2}+\alpha^{3}\right) x^{2}+(1-9 \alpha \\
& \left.+6 \alpha^{2}-2 \alpha^{3}\right) x^{3}+\left(-2-\alpha+3 \alpha^{2}-\alpha^{3}\right) x^{4} \\
& +\left(-2+4 \alpha-3 \alpha^{2}+\alpha^{3}\right) x^{5}
\end{aligned}
$$

The ranges of $(P, \alpha)$ described in expression 8 , for which the 2-clique network exhibits a turning point, are shown in the filled area in Fig. 3.

\section{Conclusions}

We analyze the effect of self-generated information-i.e., information created at the node level by a node that is not informed-on the dynamics of rumor spread in a network. Our findings suggest that the transmission of self-generated signals (in addition to received signals) does not necessarily make a network more active. We analyze whether the transmission of self-generated signals leads to development of a turning point, meaning a non-monotonic effect on rumor 
spread time (the time required for a predefined proportion of the nodes to become informed), due to suspicion arising from the presence of bullshit information in the network.

In the numerical case, we analyze various network setups and show that increasing the internal transmission of self-generated signals may have both positive and negative effects on the rumor spread time when both of these two conditions hold: when the network is sparsely connected, and when other internal communication rate (non-self-generated) is low.

Analytically, we achieve a general formula for the probability of adding new informed nodes in a general clique, which shows the complexity of fully exploring the model analytically. We further analyze the case of a 2-clique network, showing that a turning point may or may not exist, depending on the model parameters.

Acknowledgements We thank Dr. Ilan Gronau of the Interdisciplinary Center Computer Science School for his knowledgeable and useful remarks.

Data and code availability The datasets generated and/or analyzed for the current study are available in a Mendeley repository (Tuchner and Gilboa-Freedman 2020b). The codebase used for this study is available in GitHub. This includes the Matlab code for conducting the computational simulations, and the Python code for building a decision tree based on the output of the simulations (Tuchner and Gilboa-Freedman 2020a, c), as well as the Wolfram Mathematica code for analytical examination of the 2-clique version of the model (Tuchner and GilboaFreedman 2020d)

\section{References}

Allcott H, Matthew G (2017) Social media and fake news in the 2016 election. J Econ Perspect 31:211-236

Bikhchandani S, Hirshleifer D, Welch I (1992) A theory of fads, fashion, custom, and cultural change as informational cascades. J Political Econ 100:992-1026

Budak C, Agrawal D, El Abbadi A (2011) Limiting the spread of misinformation in social networks. In: Proceedings of the 20th international conference on World Wide Web, pp 665-674

Conroy NJ, Rubin VL, Chen Y (2015) Automatic deception detection: methods for finding fake news. In: Proceedings of the 78th ASIS\&T annual meeting, $\mathrm{p} 82$

Cuypere ED, Koen DT, Wittevrongel S, Fiems D (2016) Opinion propagation in bounded medium-sized populations. Perform Eval 99:1-15

Delen D, Kuzey C, Uyar A (2013) Measuring firm performance using financial ratios: a decision tree approach. Expert Syst Appl 40(10):3970-3983

Dhar J, Jain A, Gupta VK (2016) A mathematical model of news propagation on online social network and a control strategy for rumor spreading. Soc Netw Anal Min 6(1):57

Fan L, Wu W, Zhai X, Xing K, Lee W, Du DZ (2014) Maximizing rumor containment in social networks with constrained time. Soc Netw Anal Min 4(1):214

Fehr E, Fischbacher U (2003) The nature of human altruism. Nature 425:785-791
Frankfurt HG, Wilson G (2005) On bullshit. Princeton University Press, Princeton

Gilboa-Freedman G, Smorodinsky R (2020) On the properties that characterize privacy. Math Soc Sci 103:59-68

Girvan M, Newman MEJ (2002) Community structure in social and biological networks. In: Proceedings of the national academy of sciences, pp 7821-7826

Goldenberg J, Libai B, Muller E (2001) Talk of the network: a complex systems look at the underlying process of word-of-mouth. Mark Lett 12:211-223

Gottfried J, Shearer E (2016) News use across social media platforms 2016. Pew Research Center. http://www.journalism .org/2016/05/26/news-use-across-social-media-platforms-2016/

Gupta A, Lamba H, Kumaraguru P, Joshi A (2013) Faking Sandy: characterizing and identifying fake images on Twitter during Hurricane Sandy. In: Proceedings of the 22nd international conference on World Wide Web (WWW'13 Companion). ACM, New York, pp 729-736

Habib A, Asghar MZ, Khan A, Habib A, Khan A (2019) False information detection in online content and its role in decision making: a systematic literature review. Soc Netw Anal Min 9(1):50

Hayes B, Kotwica K (2013) Crisis management at the speed of the internet: trend report. Elsevier, Amsterdam

Holland JH (1995) Hidden order: how adaptation builds complexity. Addison-Wesley, New York

Jurvetson S (2000) What exactly is viral marketing. Red Herring 78:110-112

Kempe D, Kleinberg JM, Tardos E (2003) Maximizing the spread of influence through a social network. In: Proceedings of the ninth ACM SIGKDD international conference on knowledge discovery and data mining, pp 137-146

Keyes R (2004) The post-truth era: dishonesty and deception in contemporary life. St. Martin's Press, New York

Koren H, Kaminer I, Raban DR (2014) Exploring the effect of reinvention on critical mass formation and the diffusion of information in a social network. Soc Netw Anal Min 4(1):185

Kotsiantis SB, Zaharakis I, Pintelas P (2007) Supervised machine learning: a review of classification techniques. Emerg Artif Intell Appl Comput Eng 160:3-24

Krider RE, Weinberg CB (1998) Competitive dynamics and the introduction of new products: the motion picture timing game. J Mark Res 35:1-15

Kucharski A (2016) Post-truth: study epidemiology of fake news. Nature 540:525

Lazer DMJ, Baum MA et al (2018) The science of fake news. Science 359(6380):1094-1096

Leskovec J (2012) Social circles: Facebook. SNAP Project, Stanford University

Liggett TM (2012) Interacting particle systems, vol 276. Springer, Berlin

Marchi R (2012) With Facebook, blogs, and fake news, teens reject journalistic "objectivity". J Commun Inq 36:246-262

Mendoza M, Poblete B, Castillo C (2010) Twitter under crisis: can we trust what we RT? In: Proceedings of the first workshop on social media analytics (SOMA '10). ACM, New York, pp 71-79

Quinlan J (1986) Induction of decision trees. Mach Learn 1(1):81-106

Safavian SR, Landgrebe D (1991) A survey of decision tree classifier methodology. IEEE Trans Syst Man Cybern 21(30):660-674

Savage LJ (1951) The theory of statistical decision. J Am Stat Assoc 46:55-67

Shannon CE (1948) A mathematical theory of communication. Bell Syst Tech J 27:379-423

Simon HA (1960) The new science of management decision. Harper \& Brothers, New York

Starbird K, Maddock J, Orand M, Achterman P, Mason R (2014) Rumors, false flags, and digital vigilantes: misinformation on 
Twitter after the 2013 Boston Marathon Bombing. In: iConference 2014 proceedings, pp 654-662

Tuchner T, Gilboa-Freedman G (2019) Crying "wolf"in a network structure: the influence of node-generated signals. In: International conference on complex networks and their applications. Springer, pp 301-312

Tuchner T, Gilboa-Freedman G (2020a) Decision tree turning point existence. https://github.com/tomertuc/DecisionTreeTurningP ointExistence

Tuchner T, Gilboa-Freedman G (2020b) Rumor spread with self-generated signals-extended simulation results. https://data.mende ley.com/datasets/w24mmm27f9/1

Tuchner T, Gilboa-Freedman G (2020c) Self-generated signals. https ://github.com/tomertuc/SelfGeneratedSignals

Tuchner T, Gilboa-Freedman G (2020d) Turning point in a two-node network. https://github.com/tomertuc/TurningPointInTwoNod esNetwork
Vosoughi S, Roy D, Aral S (2018) The spread of true and false news online. Science 359(6380):1146-1151

Waldrop M (1993) Complexity: the emerging science at the edge of order and chaos. Simon and Schuster, New York

Wang L, Wang Y, De Melo G, Weikum G (2019) Understanding archetypes of fake news via fine-grained classification. Soc Netw Anal Min 9(1):37

Wasserman S, Faust K (1994) Social network analysis: methods and applications, vol 8. Cambridge University Press, Cambridge

Watts DJ, Strogatz SH (1998) Collective dynamics of "small-world" networks. Nature 393:440-442

Wedgwood R (2002) The aim of belief. Nous 36(s16):267-297

Publisher's Note Springer Nature remains neutral with regard to jurisdictional claims in published maps and institutional affiliations. 